\title{
Objetos de Aprendizagem no Apoio à Aprendizagem de Engenharia: Explorando a Motivação Extrínseca
}

Alberto Bastos do Canto Filho, PGIE - UFRGS, alberto.canto@ufrgs.br

Luiz Fernando Ferreira, Engenharia Elétrica - UFRGS, luff.ferreira@ gmail.com

Magda Bercht - PGIE / UFRGS, bercht@inf.ufrgs.br

Liane Margarida Rockenbach Tarouco, PGIE/ UFRGS, liane@ penta.ufrgs.br

José Valdeni de Lima, PGIE/UFRGS, valdeni@inf.ufrgs.br

\begin{abstract}
Resumo.
Este artigo propõe um método de apoio à aprendizagem que utiliza Objetos de Aprendizagem para melhorar o rendimento de estudantes de engenharia. A proposta explora os recursos tecnológicos para automatizar práticas que, embora viáveis em aulas particulares, são impraticáveis no contexto típico de cursos de engenharia: aulas expositivas ministradas para turmas com um grande número de alunos. É dada ênfase ao domínio afetivo, explorando-se a motivação extrínseca dos estudantes para modificar seus hábitos de estudo. Apresenta-se também um estudo de caso, comparando o método proposto com o método de ensino e aprendizagem tipicamente utilizado.
\end{abstract}

\section{Palavras Chave: motivação, objeto de aprendizagem, dimensão afetiva.}

\section{Abstract :}

This paper proposes a teaching/learning method intended to increment the effectiveness of engineering courses. The proposed method is based on Learning Objects using technological resources to achieve a personalized teaching. The extrinsic motivation is stressed to change common study practices and improve student learning. A case study is also presented, comparing the proposed method with the traditional process of expositive classes.

\section{Keywords: motivation, learning object, affective dimension}

\section{Introdução}

O crescimento econômico do Brasil nos últimos anos trouxe consigo uma forte demanda por profissionais da área de engenharia. A expressão Apagão da Engenharia, passou a ser utilizada devido à preocupação com a possibilidade de faltarem engenheiros para realizar os projetos necessários para dar sustentação a este crescimento econômico. (Figura 1, ABRIL, 2009; EPOCA, 2011; IG, 2010; IPEA, 2010; VEJA, 2009).

O incremento do número de profissionais de nível superior em geral e de engenheiros em particular foi abordado através do programa REUNI, de expansão e reestruturação das universidades federais, que propiciou um incremento de 14.826 vagas no ensino superior, entre 2007 e 2008 (MEC, 2008). Na Escola de Engenharia da Universidade Federal do Rio Grande do Sul, entre os cursos atendidos pelo Departamento de Engenharia Elétrica, partiu-se de um cenário de 130 vagas oferecidas para ingressantes em cursos de Engenharia Elétrica e Engenharia da Computação (2007), para uma nova situação em que se criaram os cursos de Engenharia de Controle e Automação e Engenharia de Energia, chegando-se à oferta de 200 vagas em 2010. 


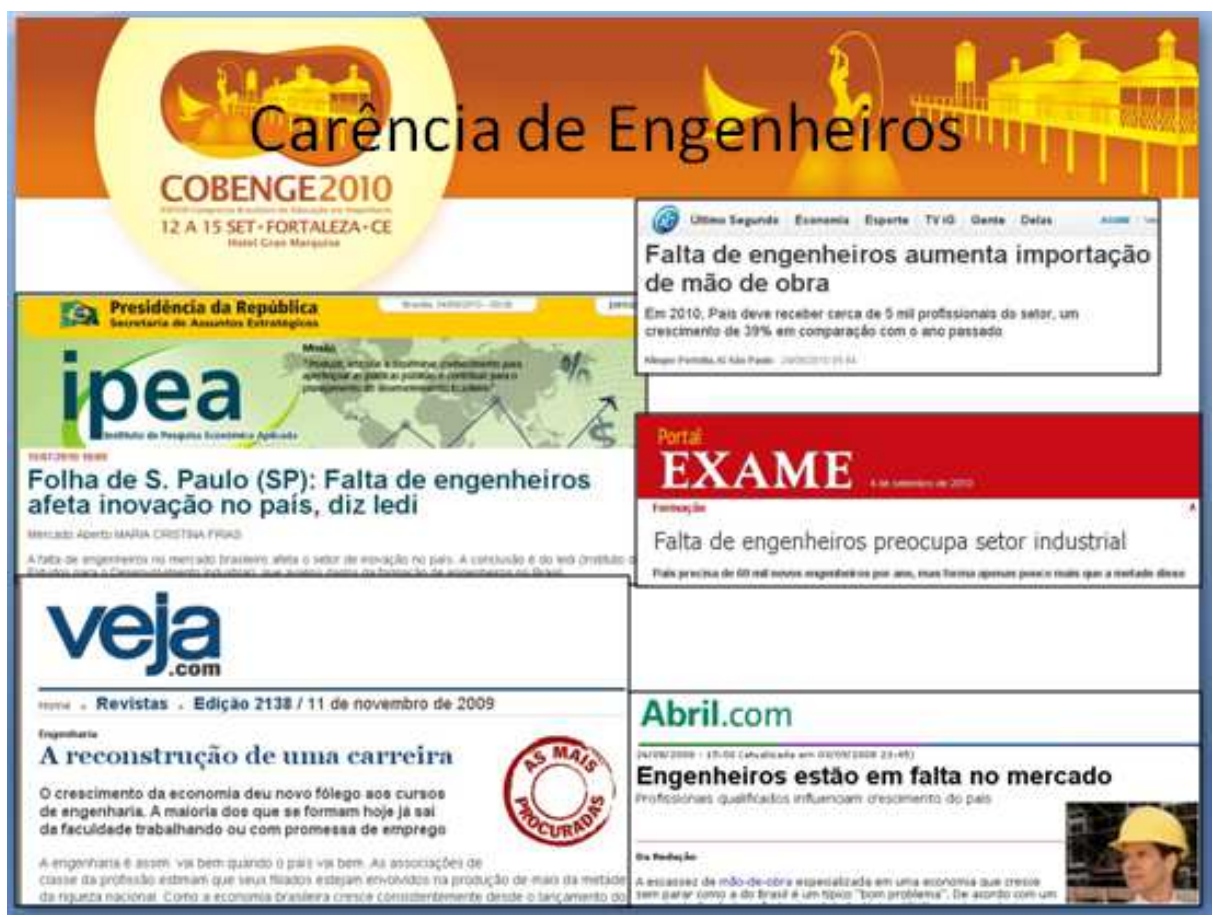

Figura 1: Carência de Engenheiros no Brasil (CANTO et al, 2011)

Infelizmente este aumento do número de vagas não foi acompanhado pelo aumento de interesse pela carreira de engenharia, ocasionando uma queda de 8,11 candidatos/vaga em 2007 para 6,3 candidatos/vaga em 2010. Esta queda, associada ao sistema de ingresso por cotas, fez com que no período 2007-2010 triplicasse o número de ingressantes com argumento de ingresso no vestibular inferior a 550. Isto é, corre-se o risco de que o expressivo aumento do número de vagas para ingressantes tenha resultado irrelevante em termos de concluintes, pois a maioria dos novos ingressantes possuem lacunas de formação, uma das principais causas que dificultam a trajetória dos estudantes nos cursos de engenharia, especialmente nas disciplinas iniciais de matemática e física (LODER, 2009). O Gráfico 1 mostra como evidência deste fato, o crescimento dos percentuais de reprovação na disciplina de Física I.

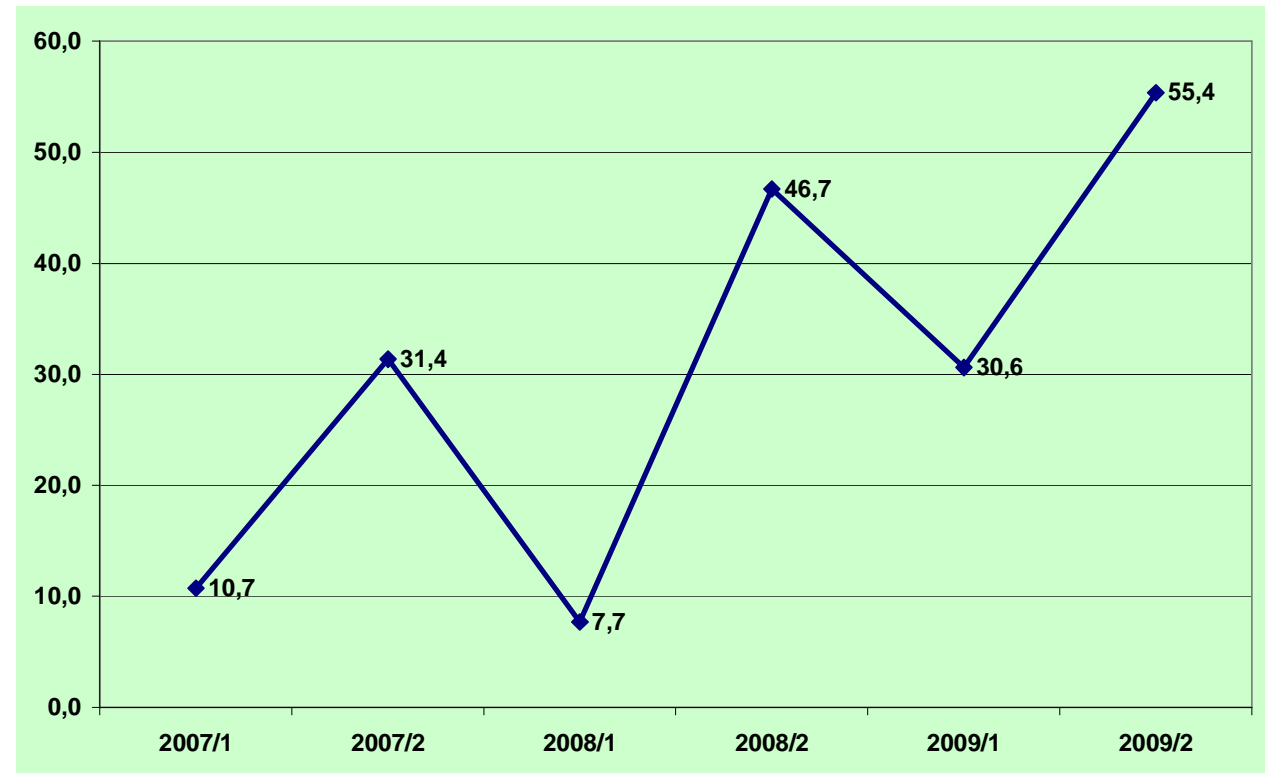

Gráfico 1: Reprovação na disciplina de Física I (CANTO et al, 2011) 
O ingresso de um percentual maior de estudantes cuja mensuração de desempenho indica lacunas formativas em áreas essenciais e o aumento dos índices de reprovação nas cadeiras iniciais resulta não apenas no aumento dos índices de evasão, mas também numa maior demanda de vagas para atender os alunos retidos, o representa uma necessidade de mais espaço físico, docentes e recursos. Neste contexto, surge a questão de pesquisa enunciada no Quadro 1.

É possível utilizar Tecnologia de Informação e Comunicação (TIC) para reduzir a evasão dos cursos de engenharia, sem redução de qualidade, sem incremento de horas docentes trabalhadas e sem aumentar o espaço físico ocupado?

\section{Quadro 1: Questão de Pesquisa}

A falta de clareza quanto aos potenciais de uso de Tecnologia de Informação e Comunicação (TICs) nos processos educacionais (PARRISH, 2004), é uma consequência natural do fato de que os resultados não dependem apenas do uso de TICs, mas sim da adequação dos métodos e recursos tecnológicos aos objetivos educacionais e às características dos estudantes.

Neste artigo é apresentada uma proposta de método de apoio à aprendizagem que explora Objetos de Aprendizagem (OAs) para alcançar objetivos educacionais estabelecidos dentro dos domínios cognitivo e afetivo (BLOOM, 1956). É apresentado um estudo de caso em que o método proposto é comparado com o método tradicional de apresentação de conteúdos através de aulas expositivas ministradas para turmas com um grande número de alunos.

\section{Personalização do Ensino e Princípios de Projeto}

Aulas tradicionais expositivas, ministradas para um grande número de alunos diferem significativamente de aulas particulares. Bloom (1984) identificou que alunos que receberam tutoria individual obtiveram um resultado médio de dois desvios padrões (dois sigma) acima do resultado médio alcançado por estudantes que participaram de aulas expositivas ministradas para trinta estudantes. Esta significativa diferença deve-se ao fato de que a tutoria individual possibilita níveis de personalização e interação impossíveis de serem alcançadas nos sistemas tradicionais, baseados aulas expositivas ministradas para turmas grandes. Infelizmente, os elevados custos da tutoria individual impedem que esta alternativa seja adotada em larga escala.

$\mathrm{Na}$ proposta aqui apresentada, assume-se que Objetos de Aprendizagem podem incorporar algumas práticas da tutoria individual, de forma a obter uma maior eficácia educacional ${ }^{1}$ sem que seja necessário arcar com os elevados custos da tutoria individual.

Características individuais de aprendizagem: são todas as características individuais e fatores circunstanciais que definem a forma como o estudante aprende num determinado momento. Incluem fatores tais o seu estilo de aprendizagem, os seus esquemas cognitivos, a sua memória de trabalho a quantidade e qualidade de subsunçores, seus métodos de estudos, sua velocidade de aprendizagem, sua motivação, bem como fatores ambientais e circunstanciais, tais como a disponibilidade de computador e banda larga, distância de residência até a sala de aula, horário das aulas, disponibilidade de tempo e local para estudo extraclasse, condição socioeconômica, etc.

\section{Quadro 2: Características individuais de aprendizagem}

Neste contexto, a expressão personalização do ensino é utilizada com sentido de desenvolver um OA que concilie os recursos oferecidos pela tecnologia da informação

${ }^{1}$ Eficácia educacional: é proporção em que os objetivos são alcançados (Fundamentado na definição NBR 9241-11) 
com os objetivos educacionais e com as características individuais de aprendizagem (vide definição no Quadro 2 acima).

O projeto de Objetos de Aprendizagem poderá ser concebido a partir de premissas que ponderam as características individuais diferenciadoras dos estudantes, de premissas sobre características comuns aos estudantes, ou premissas sobre características que não podem ser contempladas no projeto do OA. Alguns princípios de projeto que explorem as características de aprendizagem podem também ser aplicados em aulas expositivas presenciais, como por exemplo, a proposta de Moreno e Mayer (2007) de utilização do sentido da audição para abordar informações verbais e o sentido da visão para apresentar informações não verbais (multimídia e multimodal). Outras possibilidades pedagógicas, como a interatividade, podem ser aplicadas à modalidade presencial em situações de tutoria individual ou OAs, mas possuem restrições progressivas à medida que cresce o número de alunos por turma. Nesta seção serão apresentados princípios de projeto utilizados no estudo de caso relatado, que fundamentam o método de apoio à aprendizagem proposto.

\subsection{Princípio dos Subsunçores}

O princípio dos subsunçores aqui proposto como um princípio de projeto de OAs é um postulado da Teoria do Aprendizado Significativo de Ausubel (1980):

Princípio dos Subsunçores: Objetos de Aprendizagem são mais eficazes quando aplicados a estudantes que possuam subsunçores em quantidade e qualidade adequados aos objetivos educacionais estabelecidos.

$\mathrm{O}$ projeto de um OA pode assumir que todos os estudantes possuem os subsunçores necessários para alcançar os objetivos educacionais propostos pelo OA. Neste caso nenhum nível de personalização destas diferenças individuais é incorporado.

Alternativamente, esta relevante característica individual poderá ser tratada de forma personalizada, conforme propõe a Figura 2. Para tanto é necessário que, na etapa de especificação e projeto (Figura 2a), se estabeleçam os objetivos educacionais, seus pré-requisitos, e se especifiquem os testes de pré-requisitos. Ao ser executado (Figura 2b), o OA realiza o teste para verificar se o estudante possui os pré-requisitos necessários. Caso o estudante não possua subsunçores em quantidade e qualidade adequados, é feito um redirecionamento para o aprendizado destes conteúdos preliminares. Observe que este procedimento de personalização do ensino é normalmente realizado em tutorias individuais, e pode ser sistematizado para aplicação em larga escala, quando incorporado ao projeto de OAs.

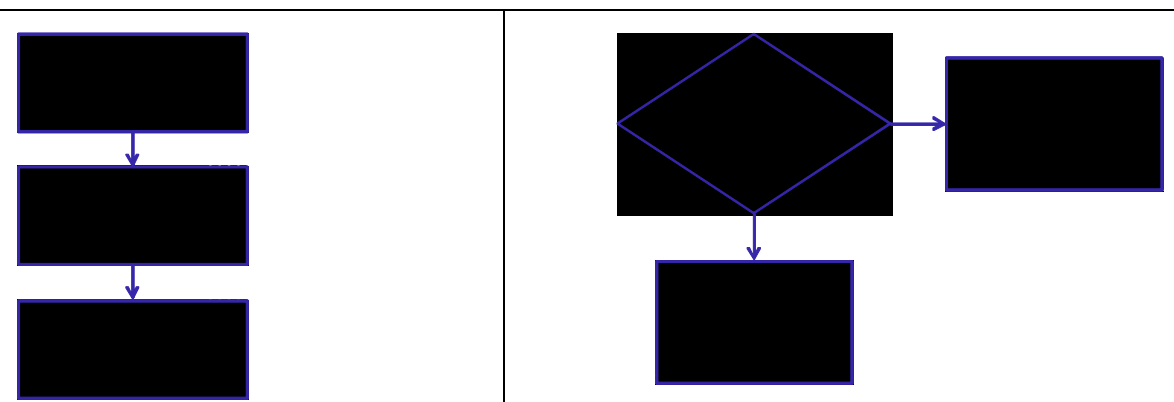

Figura 2a. Especificação

Figura 2b. Execução

Figura 2: Fluxogramas de especificação e execução de OAs 


\subsection{Princípio da Dimensão Afetiva}

Diversos autores (Mayer, 2005; Moreno e Mayer, 2007; Merriboer \& Sweller, 2010) tem apresentado recomendações de projeto de Objetos de Aprendizagem fundamentadas em princípios cognitivos (Vide seção 2.3 abaixo). Não obstante a relevância dos aspectos cognitivos, o designer instrucional não poderá se preocupar exclusivamente com os aspectos cognitivos. Pelo contrário, a ponderação da dimensão afetiva é um princípio fundamental, pois o estudante somente aprenderá se estiver adequadamente motivado:

Princípio da dimensão afetiva: Objetos de Aprendizagem especificados com uma perspectiva afetiva são mais eficazes do que aqueles que não o são.

As investigações de Kindt et al (2011) e de Baeten et al, (2010) estabelecem relações entre a motivação autônoma (alto grau de autodeterminação) a complexidade das tarefas, e a profundidade de abordagem para o aprendizado ${ }^{2}$. Ao projetar um OA que tenha por objetivo trabalhar com a motivação para incentivar uma abordagem profunda de aprendizado, o projetista do OA deve ter presente que os estudantes intrinsecamente motivados não devem ser o foco de preocupação, pois estes estudantes já estão autodeterminados para alcançar o objetivo educacional. Por outro lado, para o atendimento de estudantes que não estejam intrinsecamente motivados, faz-se necessária a identificação de fatores motivacionais extrínsecos, capazes de proporcionar o maior grau de autodeterminação ${ }^{3}$ possível. Uma alternativa simples que pode ser utilizada para implementação desta alternativa é o aproveitamento da experiência adquirida nas aulas presenciais, que possibilita ao docente conhecer as motivações dos estudantes e utilizá-las em favor de um aprendizado mais eficaz.

\subsection{Princípios de Projeto e Carga Cognitiva}

Diversos autores (Mayer, 2005; Mayer e Moreno, 2003; Moreno e Mayer, 2007; Van Merriboer e Sweller, 2010) apresentam princípios de projeto que podem ser fundamentados na estrutura cognitiva humana. Van Merriboer e Sweller (2010) apresentam esta arquitetura, considerando a existência de dois tipos de memória: memória de longo prazo e memória de trabalho. A memória de longo prazo possui capacidade virtualmente infinita, mas não pode ser acessada conscientemente pelo ser humano; os processos conscientes fazem uso de uma memória de trabalho, que armazena elementos de informação trazidos da memória de longo prazo e elementos de informação novos, adquiridos através da memória sensorial (sentidos). Esta memória de trabalho possui dois tipos de limitação: a. limitação do número máximo de elementos de informação simultâneos: tipicamente, o ser humano consegue lidar em sua memória de trabalho com cinco a nove elementos de informação simultaneamente (sete mais ou menos dois). b. Limitação temporal: elementos novos de informação, que são perdidos cerca de 20 segundos após o último acesso. A teoria Baddeley (1992) enfatizou e existência de canais independentes de processamento de informações, associados aos modos sensoriais individuais: um canal para lidar com esquemas visuais ou espaciais e outro canal para lidar com as informações verbais e auditivas. $\mathrm{O}$ uso destes dois canais

${ }^{2}$ Kyndt et al, (2011) definem "Abordagem profunda para o aprendizado"como aquela em que o estudante empenha um esforço sincero e eficaz para o entendimento, adotando estratégias tais como a busca de relacionamento dos novos conteúdos com seus conhecimentos existentes, reflexão, verificação de evidências, exame crítico dos argumentos, discussões, busca de informações complementares, etc..

${ }^{3}$ Ryan e Deci (2000) propõem uma escala de autodeterminação crescente conforme o tipo de controle da motivação extrínseca. Nas escalas mais baixas estão as motivações com controle externo, quando o estudante percebe que suas ações são resultado de pressão ou coerção externa. Nas escalas mais altas, com autodeterminação equivalente à motivação intrínseca, está o controle integrado aos valores e interesses do próprio estudante; a motivação extrínseca com controle integrado difere da motivação intrínseca apenas pelo fato de que os resultados podem ser separados da satisfação inerente ao aprendizado do tema. 
possibilita o tratamento de um número maior de elementos de informação simultâneos, o que está em consonância com as propostas de multimodalidade (Moreno e Mayer, 2007) e com o princípio de projeto multimídia (Mayer, 2005). O princípio na continuidade temporal (Mayer, 2005), está em consonância com a característica volátil da memória de trabalho (elementos novos de informação são perdidos cerca de 20 s após o último acesso). Outros princípios estão relacionados ao número máximo de elementos simultâneos e à capacidade de processamento decorrente. Por exemplo, os princípios da Coerência e da Continuidade Espacial (Mayer, 2005) estão alinhados com as recomendações de Van Merriboen e Sweller (2010) referentes à redução da carga cognitiva dispersiva (extraneous load), e tem por objetivo reduzir o número de elementos simultâneos na memória de trabalho.

Com o objetivo permitir que o estudante tenha tempo de integrar e organizar as imagens e palavras antes que um novo elemento de informação seja apresentado, Mayer e Moreno (2003) apresentam recomendações de segmentação e controle de ritmo em sistemas multimodais. $\mathrm{O}$ ajuste de ritmo de apresentação ao ritmo de aprendizagem do estudante é uma recomendação digna de destaque pela sua facilidade de implementação e pelo potencial ganho de eficácia de aprendizagem. Destaca-se aqui que este ajuste de ritmo não possui ganhos apenas em sistemas multimodais, pois a integração e organização são necessárias em qualquer situação de aprendizado significativo, onde estudantes necessitam de tempos diferentes para ancorar os novos elementos de informação aos seus subsunçores.

\section{Estudo de Caso}

Nesta seção será apresentado um estudo de caso realizado com o objetivo de investigar alternativas de ensino e aprendizagem que possibilitem redução de índices de evasão em cursos de engenharia. Assumiu-se que uma das principais causas da evasão é a falta de ajuste entre os métodos normalmente adotados e as características individuais dos estudantes que evadem do curso. Utilizou-se um OA desenvolvido de acordo com os princípios apresentados na seção 2, sobre o tema "Sistemas Numéricos, Números Binários", normalmente apresentado em uma aula teórica expositiva de 90 minutos, para turmas com 48 alunos ou mais. Neste estudo, os estudantes foram aleatoriamente divididos em dois grupos: Grupo OBJ e Grupo PRS. Os integrantes do grupo OBJ participaram do método proposto, acessando o $\mathrm{OA}$ e não participando da aula expositiva. Os estudantes do grupo PRS assistiram à aula expositiva tradicional e somente tiveram acesso ao OA após o teste, que envolveu os dois grupos e foi realizado na aula presencial imediatamente seguinte à aula expositiva assistida pelo grupo PRS. O objetivo deste teste foi comparar o desempenho dos dois grupos. Foi aplicado também um questionário e pesquisa de opinião.

$\mathrm{O}$ estudo de caso foi dividido em duas fases: Fase I, realizada no primeiro semestre de 2012; Fase II, realizada no segundo semestre de 2012. A seguir serão apresentadas estas duas fases.

\subsection{Estudo de Caso - Fase I (2012/1)}

Nesta primeira fase, participaram 66 estudantes dos Cursos de Engenharia Elétrica e Engenharia de Controle e Automação. Os estudantes não foram comunicados sobre o teste que seria realizado.

A análise de resultados considerou também outra segmentação, baseada no I3, indicador que correspondente ao conceito médio de cada estudante no curso. Estudantes com conceito médio A ou B, foram considerados ajustados aos métodos de ensino e aprendizagem normalmente utilizados no curso (classe ADJ). Estudantes com conceito 
médio $\mathrm{C}$ ou inferior, foram considerados não ajustados (N_ADJ). A Tabela 1 apresenta os resultados obtidos na Fase I, classificados conforme o método de ensino e aprendizagem (Grupos OBJ e PRS) e conforme o ajuste do estudante aos métodos de ensino e aprendizagem do curso (Grupos ADJ e N_ ADJ). A medição de desempenho refere-se à nota obtida no teste de conhecimentos; No grupo PRS, a medição de frequência refere-se ao percentual de alunos presentes na aula expositiva e realizaram o teste; no grupo OBJ, a medição de frequência refere-se ao percentual de estudantes que acessou o OA disponibilizado no Moodle e realizaram o teste.

Tabela 1: Resultados - Fase I

\begin{tabular}{cc|cccc|cc}
\hline & \multicolumn{3}{c|}{ Desempenho } & \multicolumn{2}{l}{ Frequência } \\
\cline { 2 - 7 } & \multicolumn{2}{c}{ OBJ } & \multicolumn{2}{c}{ PRS } & & \\
\cline { 2 - 7 } & Média & $\begin{array}{c}\text { Desvio } \\
\text { Padrão }\end{array}$ & Média & $\begin{array}{c}\text { Desvio } \\
\text { Padrão }\end{array}$ & OBJ & PRS \\
\hline Ajuste & ADJ & 9,3 & 0,9 & 8,4 & 1,5 & $78 \%$ & $76 \%$ \\
& N_ADJ & 7,8 & 2,9 & 5 & 3,7 & $48 \%$ & $38 \%$ \\
\hline
\end{tabular}

Os resultados apresentados na Tabela 1 mostram uma relevante diferença nos valores dos indicadores apurados. Comparando o grupo ADJ (ajustados aos métodos de ensino e aprendizagem do curso) com o grupo N_ADJ, observa que o grupo ADJ obteve um desempenho médio superior (médias mais elevadas) e mais homogêneo (desvio padrão menor). Também os indicadores de frequência foram superiores. Comparando o grupo OBJ com o Grupo PRS, observa-se que o desempenho médio dos estudantes do grupo OBJ foi superior ao do grupo PRS.

Os resultados apresentados indicaram que: a. os estudantes do grupo ADJ não tiveram dificuldades no aprendizado com uso do OA; b. Os estudantes do grupo N_ADJ que utilizaram o $\mathrm{OA}$ tiveram um desempenho significativamente superior ao desempenho dos estudantes que assistiram à aula expositiva (effect size de 0.93). c. Estudantes do grupo N_ADJ tiveram índices de frequência inferiores aos índices de frequência do grupo ADJ.

Estes resultados levaram à formulação de novas questões, que foram investigadas na fase II do estudo de caso: Baixos índices de frequência indicam pouca motivação intrínseca; Se o método de ensino e aprendizagem contemplasse um fator de motivação extrínseca melhoraria a frequência? E o desempenho? A medição de desempenho foi influenciada pelo fato de alguns alunos possuírem conhecimentos prévios sobre o tema abordado?

\subsection{Estudo de Caso - Fase II (2012/2)}

Nesta segunda fase do estudo do caso, introduziu-se um fator de motivação extrínseca: os estudantes foram informados sobre o teste e a avaliação de desempenho foi incluída com um peso de 15\% na segunda prova da disciplina: $10 \%$ o teste de desempenho, $3 \%$ os resultados apurados nos testes do OA, e 2\% pela participação no questionário e pesquisa de opinião. Os estudantes do grupo PRS também executaram o OA, após a realização do teste. Esta etapa do estudo de caso envolveu 47 alunos. Em função dos resultados obtidos na fase I, a investigação concentrou-se na análise do grupo N_ADJ, dos estudantes não ajustados aos métodos de ensino e aprendizagem do curso. No questionário, foram introduzidas questões sobre motivação, hábitos de estudo, e conhecimento prévio do tema abordado. O objeto utilizado foi o mesmo utilizado na fase I; o procedimento de divisão nos grupos OBJ e PRS e o teste aplicado também foram os mesmos da fase $\mathrm{I}$. 
A Tabela 2 ao lado mostra a frequência dos estudantes do grupo N_ADJ. Observe que na fase II, em que a atividade "valia nota", houve um significativo aumento de frequência. Observe também a frequência maior do grupo OBJ, o que pode ser justificada pela facilidade de poder trabalhar a qualquer hora, em qualquer lugar.

A Tabela 3 ao lado mostra o desempenho de 26 estudantes do grupo N_ADJ. Nesta tabulação, não foram considerados os estudantes que declararam já conhecer o conteúdo. Os resultados foram moderadamente significativos (effect size de 0.31).

No questionário aplicado, a questão "Estudo diariamente, mesmo que não existam avaliações agendadas para os próximos dias" foi marcada como verdadeira por $46 \%$ dos estudantes do grupo ADJ e por 39\% dos estudantes do grupo N_ADJ.

\section{Conclusão}

Neste artigo foi apresentada uma proposta de método de apoio à aprendizagem que utiliza OAs para obtenção de uma maior eficácia educacional em cursos de engenharia. A proposta parte da premissa que OAs são capazes de obter um nível de personalização maior do que existe nos métodos tradicionais, baseados em aulas expositivas ministradas para um grande número de alunos. Esta personalização pode ser obtida através do desenvolvimento de OAs dentro de princípios de projeto propostos por diversos autores e dos princípios propostos neste artigo. Um estudo de caso foi apresentado, para o qual foi desenvolvido um objeto de aprendizado que utiliza os princípios propostos, e explora a motivação extrínseca dos alunos "por nota". Observouse que: a. tanto na modalidade presencial como na modalidade à distância, houve uma maior frequência quando a execução das tarefas era incluída na avaliação da disciplina; b. Os estudantes que participaram do grupo que utilizou o OA (grupo OBJ) tiveram desempenho e frequência superiores aos estudantes que participaram da aula presencial (grupo PRS); c. Tanto no grupo PRS como no grupo OBJ, os estudantes ajustados ao sistema tradicionalmente utilizado nos cursos de engenharia (ADJ - estudantes com conceito médio de $\mathrm{A}$ ou $\mathrm{B}$ ) tiveram um desempenho significativamente maior que os estudantes não ajustados (N_ADJ).

Dentro da problemática de redução da evasão dos cursos de engenharia, o estudo de caso apresenta uma sinalização clara de que o método proposto pode ser uma alternativa eficaz para que haja uma maior frequência e melhor desempenho por parte dos alunos não ajustados aos métodos tradicionais utilizados. Atribui-se o aumento de frequência principalmente ao aspecto motivacional de "valer nota", e em segundo lugar à facilidade de acessar o OA a qualquer hora, em qualquer lugar. Destaca-se aqui que a possibilidade de realizar uma avaliação individual "em todas as aulas" é viabilizada pelo uso da tecnologia da informação; isto é, no sistema presencial com turmas numerosas, os custos deste método seriam muito elevados devido à demanda de espaço físico e tempo para aplicação das provas, além da demanda de recursos adicionais para a correção das provas.

O fato do estudo de caso ter sido aplicado para o conteúdo abordado em uma única aula impede que se possam generalizar os resultados, havendo a necessidade de 
investigação futura que vise identificar se não haveria um declínio desta motivação caso o método fosse aplicado de forma contínua, a todo o conteúdo de uma disciplina.

O melhor desempenho dos estudantes do grupo OBJ é atribuído a diversas funcionalidades do OA, que permitem um melhor ajuste a algumas características individuais de aprendizado entre as quais se destacam: a. ajuste de ritmo: o objeto foi projetado como uma sequência de segmentos de curta duração (cerca de 20s), permitindo que o estudante faça uma reflexão antes de avançar para o próximo segmento; b. Interatividade: Além da necessária intervenção para avançar após cada segmento, são apresentadas questões cuja resposta exige reflexão sobre os conteúdos previamente abordados; c. motivação e avaliação: as questões existentes no OA são pontuadas e os pontos contabilizados no sistema de avaliação da disciplina; d. ajuste da forma de apresentação: cada segmento apresenta o conteúdo através de animações, nas quais as informações verbais são apresentadas em áudio e as informações não verbais apresentadas na tela; ao final do segmento as informações verbais são apresentadas na forma escrita; o estudante poderá "pular" o áudio se preferir que as informações verbais sejam apresentadas na tela. e. Sequenciamento: o OA é subdividido em quatro seções com objetivos educacionais distintos; na primeira vez que o OA é executado, o estudante deverá responder as questões sobre uma secção antes de que a próxima seja liberada; a partir da segunda vez, o acesso às secções já executadas pode ser realizado de forma aleatória (modo revisão).

Finalmente, destaca-se que o desenvolvimento de Objetos de Aprendizagem Multimodais e interativos, implementados em conformidade com o padrão SCORM é uma atividade demandante de recursos, e que a viabilidade econômica deste investimento dependerá da escala de utilização / reutilização deste objeto.

\section{Referências Bibliográficas}

ABRIL. Engenheiros estão em falta no mercado. 2009. Disponível em: http://www.abril.com.br/noticia/estilo/no_297271.shtml. acesso em 1/8/2012.

AUSUBEL, David; NOVAK, Joseph; HANESIAN, H. Psicologia Educacional. Rio de Janeiro: Interamericana, 1980.

BADDELEY, A. Working memory. Science 255: 556-559, 1992.

BAETEN, M., E. KYNDT, K. STRUYVEN, AND F. DOCHY. 2010. Using studentcentred learning environments to stimulate deep approaches to learning: Factors encouraging or discouraging their effectiveness. Educational Research Review 5: 243260.

BLOOM, B. S. The 2 Sigma Problem: The search for methods of group instruction as effective as one-to-one tutoring". In: Educational Researcher. [S.1.]: [s.n.], 1984. p. 416.

BLOOM, B.S. Taxonomy of educational objectives: the classification of educational goals: New York; Toronto: Longmans, Green. 1956. 207 p. Handbook I, cognitive domain.

CANTO et al. Evasão e retenção em cursos de engenharia. Loder et al, Desafios da Educação em Engenharia: vocação, formação, exercício profissional, experiências metodológicas e proposições. Blumenau: Edifurb, 2011.

EPOCA. Falta de engenheiros no Brasil pode causar atrasos nas obras do PAC, Copa e Olimpíadas. 2011. Disponível em: http://revistaepoca.globo.com/Revista/Epoca/0,EMI213475-15228,00.html. acesso em $1 / 8 / 2012$. 
IG. Falta de engenheiros aumenta importação de mão de obra. 2010. Disponível em: http://economia.ig.com.br/falta-de-engenheiros-aumenta-importacao-de-mao-deobra/n1237753706634.html. acesso em 1/8/2012.

IPEA. Falta de engenheiros afeta inovação no país. 2010. Disponível em: http://www.ipea.gov.br/portal/index.php?option=com_content\&view=article\&id=1635. acesso em 1/8/2012.

KINDT E et AL. The direct and indirect effect of motivation for learning on students' approaches to learning through the perceptions of workload and task complexity.

Higher Education Research \& Development, [n.1.], v. 30, p. 135-150, 2011

LODER L. L. O aluno de engenharia elétrica: algumas considerações sobre suas expectativas, competências e trajetórias escolares. In: CONGRESSO BRASILEIRO DE ENSINO DE ENGENHARIA, Recife, 2009.

MAYER, R. E. Introduction to multimedia learning. In: MAYER, R. E. (Ed.). The Cambridge Handbook of Multimedia Learning. New York: Cambridge University Press, 2005.

MAYER R; MORENO, R. Nine Ways to Reduce Cognitive Load in Multimedia Learning. Educational Psychologist, [n.1.], v. 38, 2003

MEC. Programa de Apoio a Planos de Reestruturação e Expansão das Universidades Federais - Reuni 2008 - Relatório de Primeiro Ano. 2008. Disponível em:

http://portal.mec.gov.br/index.php?option=com_docman\&task=doc_download\&gid=20 $\underline{69}$. acesso em 1/8/2012.

MORENO, R.; MAYER R. Interactive multimodal learning environments. Educational Psychology Review,[n.1.], v. 19, p. 309-326, 2007

NBR 9241-11. Requisitos Ergonômicos para Trabalho de Escritórios com Computadores. Rio de Janeiro, ABNT, Ago/2002.

PARRISH, P. E. The Trouble with Learning Objects. Educational technology research and development. , n. 1, v. 52, pp. 49-67, 2004.

RYAN R. M, DECI E. L. Self-determination theory and the facilitation of intrinsic motivation, social development, and well-being. Am Psychol. 55: 68-78, 2000

VAN MERRIENBOER, J. J. G; SWELLER, J. Cognitive load theory in health professional education: design principles and strategies. Medical Education, 44:85-93, 2010.

VEJA. A reconstrução de uma carreira. 2009. Disponível em: http://veja.abril.com.br/111109/reconstrucao-uma-carreira-p-174.shtml. acesso em $1 / 8 / 2012$. 